\title{
On Optimization of the Importance Weighted OWA Aggregation of Multiple Criteria*
}

\author{
Włodzimierz Ogryczak and Tomasz Śliwiński \\ Warsaw University of Technology, Institute of Control \& Computation Engineering, \\ 00-665 Warsaw, Poland \\ \{wogrycza,tsliwins\}@ia.pw.edu.pl
}

\begin{abstract}
The problem of aggregating multiple numerical criteria to form overall objective functions is of considerable importance in many disciplines. The ordered weighted averaging (OWA) aggregation, introduced by Yager, uses the weights assigned to the ordered values rather than to the specific criteria. This allows one to model various aggregation preferences, preserving simultaneously the impartiality (neutrality) with respect to the individual criteria. However, importance weighted averaging is a central task in multicriteria decision problems of many kinds. It can be achieved with the Weighted OWA (WOWA) aggregation though the importance weights make the WOWA concept much more complicated than the original OWA. We show that the WOWA aggregation with monotonic preferential weights can be reformulated in a way allowing to introduce linear programming optimization models, similar to the optimization models we developed earlier for the OWA aggregation. Computational efficiency of the proposed models is demonstrated.
\end{abstract}

\section{Introduction}

Consider a decision problem defined as an optimization problem with $m$ objective functions $f_{i}(\mathbf{x})$. They can be either maximized or minimized. When all the objective functions are maximized the problem can be written as follows:

$$
\max \left\{\left(f_{1}(\mathbf{x}), f_{2}(\mathbf{x}), \ldots, f_{m}(\mathbf{x})\right): \mathbf{x} \in \mathcal{F}\right\}
$$

where $\mathbf{x}$ denotes a vector of decision variables to be selected within the feasible set $\mathcal{F} \subset R^{q}$, of constraints under consideration and $\mathbf{f}(\mathbf{x})=\left(f_{1}(\mathbf{x}), f_{2}(\mathbf{x}), \ldots, f_{m}(\mathbf{x})\right)$ is a vector function that maps the feasible set $\mathcal{F}$ into the criterion space $R^{m}$. Model (1) only specifies that we are interested in maximization of all objective functions $f_{i}$ for $i=1,2, \ldots, m$. In order to make the multiple criteria model operational for the decision support process, one needs to assume some solution concept well adjusted to the decision maker's preferences. The solution concepts

\footnotetext{
* The research was supported by the Ministry of Science and Information Society Technologies under grant 3T11C 00527 "Models and Algorithms for Efficient and Fair Resource Allocation in Complex Systems."
} 
are defined by aggregation functions $a: R^{m} \rightarrow R$. Thus the multiple criteria problem (11) is replaced with the (scalar) maximization problem

$$
\max \{a(\mathbf{f}(\mathbf{x})): \mathbf{x} \in \mathcal{F}\}
$$

The most commonly used aggregation is based on the weighted mean where positive importance weights $p_{i}(i=1, \ldots, m)$ are allocated to several criteria

$$
A_{\mathbf{p}}(\mathbf{y})=\sum_{i=1}^{m} y_{i} p_{i}
$$

The weights are typically normalized to the total $1\left(\sum_{i=1}^{m} p_{i}=1\right)$. Due to positive weights, every optimal solution to the weighted mean aggregation (i.e. problem (2) with the aggregation function (3) ) is an efficient solution of the original multiple criteria problem. However, the weighted mean allowing to define the importance of criteria does not allow to model the decision maker's preferences regarding distribution of outcomes. The latter is crucial when aggregating (normalized) uniform achievement criteria like those used in the fuzzy optimization methodologies [19] as well as in the goal programming and the reference point approaches to the multiple criteria decision support [8]. In the stochastic problems uniform objectives may represent various possible values of the same (uncertain) outcome under several scenarios 9 .

The preference weights can be effectively introduced with the so-called Ordered Weighted Averaging (OWA) aggregation developed by Yager [15]. In the OWA aggregation the weights are assigned to the ordered values (i.e. to the smallest value, the second smallest and so on) rather than to the specific criteria. Since its introduction, the OWA aggregation has been successfully applied to many fields of decision making [1920 2. When applying the OWA aggregation to multicriteria optimization problem (1) the weighting of the ordered outcome values causes that the OWA optimization problem is nonlinear even for linear programming (LP) formulation of the original constraints and criteria. Yager [16] has shown that the nature of the nonlinearity introduced by the ordering operations allows one to convert the OWA optimization into a mixed integer programming problem. We have shown [1] that the OWA optimization with monotonic weights can be formed as a standard linear program of higher dimension.

The OWA operator allows to model various aggregation functions from the maximum through the arithmetic mean to the minimum. Thus, it enables modeling of various preferences from the optimistic to the pessimistic one. On the other hand, the OWA does not allow to allocate any importance weights to specific criteria. Actually, the weighted mean (3) cannot be expressed in terms of the OWA aggregations.

Importance weighted averaging is a central task in multicriteria decision problems of many kinds, such as selection, classification, object recognition, and information retrieval. Therefore, several attempts have been made to incorporate importance weighting into the OWA operator 18 5. Finally, Torra 12 has introduced the Weighted OWA (WOWA) aggregation as a particular case of Choquet 
integral using a distorted probability as the measure. The WOWA averaging is defined by two weighting vectors: the preferential weights $\mathbf{w}$ and the importance weights $\mathbf{p}$. It covers both the weighted means (defined with $\mathbf{p}$ ) and the OWA averages (defined with w) as special cases. Actually, the WOWA average is reduced to the weighted mean in the case of equal all the preference weights and it becomes the standard OWA average in the case of equal all the importance weights. Since its introduction, the WOWA operator has been successfully applied to many fields of decision making [14 including metadata aggregation problems 17.

In this paper we analyze solution procedures for optimization problems with the WOWA objective functions. We show that the LP formulation of the OWA optimization with monotonic preferential weights 11 can easily be extended to cover optimization of the WOWA objective with arbitrary importance weights. A special attention will be paid to multiple criteria problems (1) with linear objective functions $f_{i}(\mathbf{x})=\mathbf{c}_{i} \mathbf{x}$ and polyhedral feasible sets:

$$
\mathbf{y}=\mathbf{f}(\mathbf{x})=\mathbf{C x} \quad \text { and } \quad \mathcal{F}=\left\{\mathbf{x} \in R^{q}: \mathbf{A x}=\mathbf{b}, \quad \mathbf{x} \geqq \mathbf{0}\right\}
$$

where $\mathbf{C}$ is an $m \times q$ matrix (consisting of rows $\mathbf{c}_{i}$ ), $\mathbf{A}$ is a given $r \times q$ matrix and $\mathbf{b}=\left(b_{1}, \ldots, b_{r}\right)^{T}$ is a given RHS vector. For such problems more efficient computational models may be introduced by taking advantages of the LP duality.

The paper is organized as follows. In the next section we introduce formally the WOWA operator and derive some alternative computational formula based on the Lorenz curves. We also analyze the orness/andness properties of the WOWA operator with monotonic preferential weights. In Section 3 we introduce the LP formulations for minimization of the WOWA aggregation with decreasing preferential weights and maximization of the WOWA aggregation with increasing weights. Finally, in Section 4 we demonstrate computational efficiency of the introduced models.

\section{The Importance Weighted OWA Aggregation}

\subsection{The WOWA Operator}

Let $\mathbf{w}=\left(w_{1}, \ldots, w_{m}\right)$ be a weighting vector of dimension $m$ such that $w_{i} \geq 0$ for $i=1, \ldots, m$ and $\sum_{i=1}^{m} w_{i}=1$. The corresponding OWA aggregation of outcomes $\mathbf{y}=\left(y_{1}, \ldots, y_{m}\right)$ can be mathematically formalized as follows [15. First, we introduce the ordering map $\Theta: R^{m} \rightarrow R^{m}$ such that $\Theta(\mathbf{y})=$ $\left(\theta_{1}(\mathbf{y}), \theta_{2}(\mathbf{y}), \ldots, \theta_{m}(\mathbf{y})\right)$, where $\theta_{1}(\mathbf{y}) \geq \theta_{2}(\mathbf{y}) \geq \cdots \geq \theta_{m}(\mathbf{y})$ and there exists a permutation $\tau$ of set $I$ such that $\theta_{i}(\mathbf{y})=y_{\tau(i)}$ for $i=1, \ldots, m$. Further, we apply the weighted sum aggregation to ordered achievement vectors $\Theta(\mathbf{y})$, i.e. the OWA aggregation has the following form:

$$
A_{\mathbf{w}}(\mathbf{y})=\sum_{i=1}^{m} w_{i} \theta_{i}(\mathbf{y})
$$


The OWA aggregation (5) allows to model various aggregation functions from the maximum $\left(w_{1}=1, w_{i}=0\right.$ for $\left.i=2, \ldots, m\right)$ through the arithmetic mean $\left(w_{i}=\right.$ $1 / m$ for $i=1, \ldots, m)$ to the minimum $\left(w_{m}=1, w_{i}=0\right.$ for $\left.i=1, \ldots, m-1\right)$.

Let $\mathbf{w}=\left(w_{1}, \ldots, w_{m}\right)$ and $\mathbf{p}=\left(p_{1}, \ldots, p_{m}\right)$ be weighting vectors of dimension $m$ such that $w_{i} \geq 0$ and $p_{i} \geq 0$ for $i=1, \ldots, m$ as well as $\sum_{i=1}^{m} w_{i}=1$ and $\sum_{i=1}^{m} p_{i}=1$. The corresponding Weighted OWA aggregation of outcomes $\mathbf{y}=\left(y_{1}, \ldots, y_{m}\right)$ is defined as follows [12]:

$$
A_{\mathbf{w}, \mathbf{p}}(\mathbf{y})=\sum_{i=1}^{m} \omega_{i} \theta_{i}(\mathbf{y})
$$

where the weights $\omega_{i}$ are defined as

$$
\omega_{i}=w^{*}\left(\sum_{k \leq i} p_{\tau(k)}\right)-w^{*}\left(\sum_{k<i} p_{\tau(k)}\right)
$$

with $w^{*}$ a monotone increasing function that interpolates points $\left(\frac{i}{m}, \sum_{k \leq i} w_{k}\right)$ together with the point $(0.0)$ and $\tau$ representing the ordering permutation for $\mathbf{y}$ (i.e. $\left.y_{\tau(i)}=\theta_{i}(\mathbf{y})\right)$. Moreover, function $w^{*}$ is required to be a straight line when the point can be interpolated in this way. Due to this requirement, the WOWA aggregation covers the standard weighted mean (3) with weights $p_{i}$ as a special case of equal preference weights $\left(w_{i}=1 / m\right.$ for $\left.i=1, \ldots, m\right)$. Actually, the WOWA operator is a particular case of Choquet integral using a distorted probability as the measure 4].

Note that function $w^{*}$ can be expressed as $w^{*}(\alpha)=\int_{0}^{\alpha} g(\xi) d \xi$ where $g$ is a generation function. Let us introduce breakpoints $\beta_{i}=\sum_{k \leq i} p_{\tau(k)}$ and $\beta_{0}=0$. This allows one to express weights $\omega_{i}$ as

$$
\omega_{i}=\int_{0}^{\beta_{i}} g(\xi) d \xi-\int_{0}^{\beta_{i-1}} g(\xi) d \xi=\int_{\beta_{i-1}}^{\beta_{i}} g(\xi) d \xi
$$

and the entire WOWA aggregation as

$$
A_{\mathbf{w}, \mathbf{p}}(\mathbf{y})=\sum_{i=1}^{m} \theta_{i}(\mathbf{y}) \int_{\beta_{i-1}}^{\beta_{i}} g(\xi) d \xi=\int_{0}^{1} g(\xi) F_{\mathbf{y}}^{(-1)}(\xi) d \xi
$$

where $F_{\mathbf{y}}^{(-1)}$ is the stepwise function $F_{\mathbf{y}}^{(-1)}(\xi)=\theta_{i}(\mathbf{y})$ for $\beta_{i-1}<\xi \leq \beta_{i}$. It can also be mathematically formalized as follows. First, we introduce the leftcontinuous right tail cumulative distribution function (cdf):

$$
F_{\mathbf{y}}(d)=\sum_{i \in I} p_{i} \delta_{i}(d) \quad \text { where } \quad \delta_{i}(d)= \begin{cases}1 & \text { if } y_{i} \geq d \\ 0 & \text { otherwise }\end{cases}
$$

which for any real (outcome) value $d$ provides the measure of outcomes greater or equal to $d$. Next, we introduce the quantile function $F_{\mathbf{y}}^{(-1)}$ as the right-continuous inverse of the cumulative distribution function $F_{\mathbf{y}}$ :

$$
F_{\mathbf{y}}^{(-1)}(\xi)=\sup \left\{\eta: F_{\mathbf{y}}(\eta) \geq \xi\right\} \quad \text { for } 0<\xi \leq 1
$$


Formula (8) provides the most general expression of the WOWA aggregation allowing for expansion to continuous case. The original definition of WOWA allows one to build various interpolation functions $w^{*}[13$ thus to use different generation functions $g$ in formula (8). Let us focus our analysis on the the piecewise linear interpolation function $w^{*}$. It is the simplest form of the interpolation function. Note, however, that the piecewise linear functions may be built with various number of breakpoints, not necessarily $m$. Thus, any nonlinear function can be well approximated by a piecewise linear function with appropriate number of breakpoints. Therefore, we will consider weights vectors $\mathbf{w}$ of dimension $n$ not necessarily equal to $m$. Any such piecewise linear interpolation function $w^{*}$ can be expressed with the stepwise generation function

$$
g(\xi)=n w_{k} \quad \text { for }(k-1) / n<\xi \leq k / n, \quad k=1, \ldots, n
$$

This leads us to the following specification of formula (8):

$$
A_{\mathbf{w}, \mathbf{p}}(\mathbf{y})=\int_{0}^{1} g(\xi) F_{\mathbf{y}}^{(-1)}(\xi) d \xi=\sum_{k=1}^{n} n w_{k} \int_{(k-1) / n}^{k / n} F_{\mathbf{y}}^{(-1)}(\xi) d \xi
$$

We will treat formula (11) as a formal definition of the WOWA aggregation of $m$-dimensional outcomes $\mathbf{y}$ defined by $m$-dimensional importance weights $\mathbf{p}$ and $n$-dimensional preferential weights $\mathbf{w}$. When in (86) using the integrals from the left end rather than those on intervals one gets

$$
A_{\mathbf{w}, \mathbf{p}}(\mathbf{y})=\sum_{k=1}^{n} n w_{k}\left(L\left(\mathbf{y}, \mathbf{p}, \frac{k}{n}\right)-L\left(\mathbf{y}, \mathbf{p}, \frac{k-1}{n}\right)\right)
$$

where $L(\mathbf{y}, \mathbf{p}, \beta)$ is defined by left-tail integrating $F_{\mathbf{y}}^{(-1)}$, i.e.

$$
L(\mathbf{y}, \mathbf{p}, 0)=0 \quad \text { and } \quad L(\mathbf{y}, \mathbf{p}, \beta)=\int_{0}^{\beta} F_{\mathbf{y}}^{(-1)}(\alpha) d \alpha \quad \text { for } 0<\beta \leq 1
$$

In particular, $L(\mathbf{y}, \mathbf{p}, 1)=\int_{0}^{1} F_{\mathbf{y}}^{(-1)}(\alpha) d \alpha=A_{\mathbf{p}}(\mathbf{y})$. Graphs of functions $L(\mathbf{y}, \mathbf{p}$, $\beta)$ (with respect to $\beta$ ) take the form of concave curves, the so-called (upper) absolute Lorenz curves.

Alternatively, one may refer in formula (11) to the integrals from the right end instead of intervals getting

$$
A_{\mathbf{w}, \mathbf{p}}(\mathbf{y})=\sum_{k=1}^{n} n w_{k}\left(\bar{L}\left(\mathbf{y}, \mathbf{p}, 1-\frac{k-1}{n}\right)-\bar{L}\left(\mathbf{y}, \mathbf{p}, 1-\frac{k}{n}\right)\right)
$$

where $\bar{L}(\mathbf{y}, \mathbf{p}, \beta)$ is defined by right tail integrating $F_{\mathbf{y}}^{(-1)}$, i.e.

$$
\bar{L}(\mathbf{y}, \mathbf{p}, 0)=0 \quad \text { and } \quad \bar{L}(\mathbf{y}, \mathbf{p}, \beta)=\int_{0}^{1-\beta} F_{\mathbf{y}}^{(-1)}(1-\alpha) d \alpha \quad \text { for } 0<\beta \leq 1
$$


One may easily notice that for any $0 \leq \beta \leq 1$

$$
L(\mathbf{y}, \mathbf{p}, \beta)+\bar{L}(\mathbf{y}, \mathbf{p}, 1-\beta)=\int_{0}^{1} F_{\mathbf{y}}^{(-1)}(\alpha) d \alpha=A_{\mathbf{p}}(\mathbf{y})
$$

Hence, $\bar{L}(\mathbf{y}, \mathbf{p}, 1)=A_{\mathbf{p}}(\mathbf{y})$. Graphs of functions $\bar{L}(\mathbf{y}, \mathbf{p}, \beta)$ (with respect to $\beta$ ) take the form of convex curves, the (lower) absolute Lorenz curves.

\subsection{The Orness Measures}

The OWA aggregation may model various preferences from the optimistic (max) to the pessimistic (min). Yager [15] introduced a well appealing concept of the orness measure to characterize the OWA operators. The degree of orness associated with the OWA operator $A_{\mathbf{w}}(\mathbf{y})$ is defined as

$$
\operatorname{orness}(\mathbf{w})=\sum_{i=1}^{m} \frac{m-i}{m-1} w_{i}
$$

For the max aggregation representing the fuzzy 'or' operator with weights $\mathbf{w}=$ $(1,0, \ldots, 0)$ one gets orness $(\mathbf{w})=1$ while for the min aggregation representing the fuzzy 'and' operator with weights $\mathbf{w}=(0, \ldots, 0,1)$ one has orness $(\mathbf{w})=0$. For the average (arithmetic mean) one gets orness $((1 / m, 1 / m, \ldots, 1 / m))=1 / 2$. Actually, one may consider a complementary measure of andness defined as $\operatorname{andness}(\mathbf{w})=1-\operatorname{orness}(\mathbf{w})$. OWA aggregations with orness greater or equal $1 / 2$ are considered or-like whereas the aggregations with orness smaller or equal $1 / 2$ are treated as and-like. The former correspond to rather optimistic preferences while the latter represents rather pessimistic preferences.

The OWA aggregations with monotonic weights are either or-like or andlike. Exactly, decreasing weights $w_{1} \geq w_{2} \geq \ldots \geq w_{m}$ define an or-like OWA operator, while increasing weights $w_{1} \leq w_{2} \leq \ldots \leq w_{m}$ define an and-like OWA operator. Actually, the orness and the andness properties of the OWA operators with monotonic weights are total in the sense that they remain valid for any subaggregations defined by subsequences of their weights. Namely, for any $2 \leq k \leq m$ one gets

$$
\sum_{j=1}^{k} \frac{k-j}{k-1} w_{i_{j}} \geq \frac{1}{2} \quad \text { and } \quad \sum_{j=1}^{k} \frac{k-j}{k-1} w_{i_{j}} \leq \frac{1}{2}
$$

for the OWA operators with decreasing or increasing weights, respectively. Moreover, the weights monotonicity is necessary to achieve the above total orness and andness properties. Therefore, we will refer to the OWA aggregation with decreasing weights as the totally or-like OWA operator, and to the OWA aggregation with increasing weights as the totally and-like OWA operator.

Yager [17] proposed to define the OWA weighting vectors via the regular increasing monotone (RIM) quantifiers, which provide a dimension independent description of the aggregation. A fuzzy subset $Q$ of the real line is called a RIM 
quantifier if $Q$ is (weakly) increasing with $Q(0)=0$ and $Q(1)=1$. The OWA weights can be defined with a RIM quantifier $Q$ as $w_{i}=Q(i / m)-Q((i-1) / m)$. and the orness measure can be extended to a RIM quantifier (according to $m \rightarrow \infty)$ as follows 17 .

$$
\operatorname{orness}(Q)=\int_{0}^{1} Q(\alpha) d \alpha
$$

Thus, the orness of a RIM quantifier is equal to the area under it. The measure takes the values between 0 (achieved for $Q(1)=1$ and $Q(\alpha)=0$ for all other $\alpha$ ) and 1 (achieved for $Q(0)=1$ and $Q(\alpha)=0$ for all other $\alpha$ ). In particular, $\operatorname{orness}(Q)=1 / 2$ for $Q(\alpha)=\alpha$ which is generated by equal weights $w_{k}=1 / n$. Formula (17) allows one to define the orness of the WOWA aggregation (6) which can be viewed with the RIM quantifier $Q(\alpha)=w^{*}(\alpha)[6$. Let us consider piecewise linear function $Q=w^{*}$ defined by weights vectors $\mathbf{w}$ of dimension $n$ according to the stepwise generation function (10). One may easily notice that decreasing weights $w_{1} \geq w_{2} \geq \ldots \geq w_{n}$ generate a strictly increasing concave curve $Q(\alpha) \geq \alpha$ thus guaranteeing the or-likeness of the WOWA operator. Similarly, increasing weights $w_{1} \leq w_{2} \leq \ldots \leq w_{n}$ generate a strictly increasing convex curve $Q(\alpha) \leq \alpha$ thus guaranteeing the and-likeness of the WOWA operator. Actually, the monotonic weights generate the totally or-like and and-like operators, respectively, in the sense that

$$
\int_{0}^{1} \frac{Q(a+\alpha(b-a))-Q(a)}{Q(b)-Q(a)} d \alpha \geq \frac{1}{2} \quad \text { and } \quad \int_{0}^{1} \frac{Q(a+\alpha(b-a))-Q(a)}{Q(b)-Q(a)} d \alpha \leq \frac{1}{2}
$$

for the WOWA operators with decreasing or increasing weights, respectively.

\section{LP Models for WOWA Optimization}

\subsection{Minimization of the Totally Or-Like WOWA Aggregation}

Consider minimization of a totally or-like WOWA aggregation defined by decreasing weights $w_{1} \geq w_{2} \geq \ldots \geq w_{n}$

$$
\min \left\{A_{\mathbf{w}, \mathbf{p}}(\mathbf{y}): \mathbf{y}=\mathbf{f}(\mathbf{x}), \quad \mathbf{x} \in \mathcal{F}\right\}
$$

Note that following (12) the WOWA objective function may be expressed as

$$
A_{\mathbf{w}, \mathbf{p}}(\mathbf{y})=\sum_{k=1}^{n} n w_{k}\left(L\left(\mathbf{y}, \mathbf{p}, \frac{k}{n}\right)-L\left(\mathbf{y}, \mathbf{p}, \frac{k-1}{n}\right)\right)=\sum_{k=1}^{n} w_{k}^{\prime} L\left(\mathbf{y}, \mathbf{p}, \frac{k}{n}\right)
$$

where $w_{n}^{\prime}=n w_{n}, w_{k}^{\prime}=n\left(w_{k}-w_{k+1}\right)$ while values of function $L(\mathbf{y}, \mathbf{p}, \alpha)$ for any $0 \leq \alpha \leq 1$ can be found by optimization:

$$
L(\mathbf{y}, \mathbf{p}, \alpha)=\max _{u_{i}}\left\{\sum_{i=1}^{m} y_{i} u_{i}: \sum_{i=1}^{m} u_{i}=\alpha, \quad 0 \leq u_{i} \leq p_{i} \quad \forall i\right\}
$$


The above problem is an LP for a given outcome vector $\mathbf{y}$ while it becomes nonlinear for $\mathbf{y}$ being a vector of variables. This difficulty can be overcome by taking advantage of the LP dual to (20). Introducing dual variable $t$ corresponding to the equation $\sum_{i=1}^{m} \quad u_{i}=\alpha$ and variables $d_{i}$ corresponding to upper bounds on $u_{i}$ one gets the following LP dual of problem (20):

$$
L(\mathbf{y}, \mathbf{p}, \alpha)=\min _{t, d_{i}}\left\{\alpha t+\sum_{i=1}^{m} p_{i} d_{i}: t+d_{i} \geq y_{i}, d_{i} \geq 0 \quad \forall i\right\}
$$

Minimization of WOWA with decreasing weights results in positive values of $w_{k}^{\prime}$ and leads to the problem

$$
\min _{t_{k}, d_{i k}}\left\{\sum_{k=1}^{n} w_{k}^{\prime}\left[\frac{k}{n} t_{k}+\sum_{i=1}^{m} p_{i} d_{i k}\right]: t_{k}+d_{i k} \geq y_{i}, d_{i k} \geq 0 \quad \forall i, k\right\}
$$

While taking into account the criteria and constraints of the MOLP problem (4) we get the following LP formulation of the WOWA optimization problem (18):

$$
\begin{array}{ll} 
& \min \sum_{k=1}^{n} \frac{k}{n} w_{k}^{\prime} t_{k}+\sum_{k=1}^{n} \sum_{i=1}^{m} w_{k}^{\prime} p_{i} d_{i k} \\
\text { s.t. } & \mathbf{A} \mathbf{x}=\mathbf{b} \\
& \mathbf{y}-\mathbf{C} \mathbf{x}=\mathbf{0} \\
& d_{i k} \geq y_{i}-t_{k} \text { for } i=1, \ldots, m ; k=1, \ldots, n \\
& d_{i k} \geq 0 \text { for } i=1, \ldots, m ; k=1, \ldots, n ; x_{j} \geq 0 \forall j
\end{array}
$$

This LP problem contains $m n+m+n+q$ variables and $m n+m+r$ constraints. Thus, for not too large values of $m$ and $n$ it can be solved directly. Actually, the LP model is quite similar to that introduced in [1] for the OWA optimization (c.f., model (30)-(34)).

The number of constraints in problem (22)-(26) is similar to the number of variables. However, the crucial number of variables $\left(m n\right.$ variables $\left.d_{i k}\right)$ is associated with singleton columns. Therefore, it may be better to deal with the dual of (22) - (26) where the corresponding rows become simple upper bounds, thus reducing dramatically the LP problem size. While introducing the dual variables: $\mathbf{u}=\left(u_{1}, \ldots, u_{r}\right), \mathbf{v}=\left(v_{1}, \ldots, v_{m}\right)$ and $\mathbf{z}=\left(z_{i k}\right)_{i=1, \ldots, m ; k=1, \ldots, n}$ corresponding to the constraints (23), (24) and (25), respectively, we get the following dual:

$\max \mathbf{u b}$

$$
\begin{array}{ll}
\text { s.t. } & \mathbf{u A}-\mathbf{v} \mathbf{C} \leqq \mathbf{0} \\
& v_{i}-\sum_{k=1}^{n} z_{i k}=0 \quad \text { for } i=1, \ldots, m \\
& \sum_{i=1}^{m} z_{i k}=\frac{k}{n} w_{k}^{\prime} \quad \text { for } k=1, \ldots, n \\
0 \leq z_{i k} \leq p_{i} w_{k}^{\prime} & \text { for } i=1, \ldots, m ; k=1, \ldots, n
\end{array}
$$


The dual problem (27) is consisted of only $m+n+q$ structural constraints on $m n+r+m$ variables. Since the average complexity of the simplex method depends on the number of constraints, the dual model (27) can be directly solved for quite large values of $m$ and $n$. Moreover, the columns corresponding to $m n$ variables $z_{i k}$ form the network (node-link incidence) matrix thus allowing one to employ special techniques of the network embedded simplex algorithm [3].

\subsection{Maximization of the Totally And-Like WOWA Aggregation}

Consider now maximization of a totally and-like WOWA aggregation defined by increasing weights $w_{1} \leq w_{2} \leq \ldots \leq w_{n}$

$$
\max \left\{A_{\mathbf{w}, \mathbf{p}}(\mathbf{y}): \mathbf{y}=\mathbf{f}(\mathbf{x}), \quad \mathbf{x} \in \mathcal{F}\right\}
$$

By consideration of $\mathbf{-} \mathbf{y}$ instead of $\mathbf{y}$ the problem may be reduced to the minimization of a totally or-like WOWA aggregation defined by decreasing weights. Alternatively, taking advantages of formula (14) the WOWA objective function may be expressed as

$$
A_{\mathbf{w}, \mathbf{p}}(\mathbf{y})=\sum_{k=1}^{n} n w_{k}\left(\bar{L}\left(\mathbf{y}, \mathbf{p}, 1-\frac{k-1}{n}\right)-\bar{L}\left(\mathbf{y}, \mathbf{p}, 1-\frac{k}{n}\right)\right)=\sum_{k=1}^{n} w_{k}^{\prime \prime} \bar{L}\left(\mathbf{y}, \mathbf{p}, \frac{k}{n}\right)
$$

with weights $w_{k}^{\prime \prime}=-w_{n-k}^{\prime}=n\left(w_{n-k+1}-w_{n-k}\right)$ for $k=1, \ldots, n-1$ and $w_{n}^{\prime \prime}=n w_{1}$ while values of function $\bar{L}(\mathbf{y}, \mathbf{p}, \xi)$ for any $0 \leq \xi \leq 1$ are given by optimization:

$$
\bar{L}(\mathbf{y}, \mathbf{p}, \xi)=\min _{u_{i}}\left\{\sum_{i=1}^{m} y_{i} u_{i}: \sum_{i=1}^{m} u_{j}=\xi, \quad 0 \leq u_{i} \leq p_{i} \quad \forall i\right\}
$$

Introducing dual variable $t$ corresponding to the equation $\sum_{i=1}^{m} u_{i}=\xi$ and variables $d_{i}$ corresponding to upper bounds on $u_{i}$ one gets the following LP dual expression of $\bar{L}(\mathbf{y}, \mathbf{p}, \xi)$

$$
\bar{L}(\mathbf{y}, \mathbf{p}, \xi)=\max _{t, d_{i}}\left\{\xi t-\sum_{i=1}^{m} p_{i} d_{i}: t-d_{i} \leq y_{i}, d_{i} \geq 0 \quad \forall i\right\}
$$

Note that maximization of the WOWA with increasing weights $w_{k}$ results in problem

$$
\max \left\{\sum_{k=1}^{n} w_{k}^{\prime \prime} \bar{L}\left(\mathbf{y}, \mathbf{p}, \frac{k}{n}\right): \mathbf{y}=\mathbf{f}(\mathbf{x}), \quad \mathbf{x} \in \mathcal{F}\right\}
$$

with positive weights $w_{k}^{\prime \prime}$. Therefore, maximization of the WOWA aggregation (28) can be expressed as follows

$$
\begin{aligned}
\max _{t_{k}, d_{i k}, y_{i}, x_{j}} & \sum_{k=1}^{n} w_{k}^{\prime \prime}\left[\frac{k}{n} t_{k}-\sum_{i=1}^{m} p_{i} d_{i k}\right] \\
\text { s.t. } & t_{k}-d_{i k} \leq y_{i}, d_{i k} \geq 0 \quad \text { for } i=1, \ldots, m ; k=1, \ldots, n \\
& \mathbf{y} \leq \mathbf{f}(\mathbf{x}), \quad \mathbf{x} \in \mathcal{F}
\end{aligned}
$$


In the case of MOLP model (4) this leads us to the following LP formulation of the WOWA maximization problem (28):

$$
\begin{array}{ll} 
& \max \sum_{k=1}^{n} \frac{k}{n} w_{k}^{\prime \prime} t_{k}-\sum_{k=1}^{n} \sum_{i=1}^{m} w_{k}^{\prime \prime} p_{i} d_{i k} \\
\text { s.t. } & \mathbf{A} \mathbf{x}=\mathbf{b} \\
& \mathbf{y}-\mathbf{C} \mathbf{x}=\mathbf{0} \\
& d_{i k} \geq t_{k}-y_{i} \text { for } i=1, \ldots, m ; k=1, \ldots, n \\
& d_{i k} \geq 0 \text { for } i=1, \ldots, m ; k=1, \ldots, n ; x_{j} \geq 0 \forall j
\end{array}
$$

The problem has the identical structure as that of (22)-(26) differing only with some negative signs in the objective function (32) and the deviation variable definition (35). While in (22)-(26) variables $d_{i k}$ represent the upperside deviations from the corresponding targets $t_{k}$, here they represent the downside deviations for those targets. Note that WOWA model (32)- (36) differs from the analogous deviational model for the OWA optimization [11 only due to coefficients within the objective function (32) and the possibility of different values of $m$ and $n$. In other words, the OWA deviational model [11] can easily be expanded to accommodate the importance weighting of WOWA.

Model (32) -(36) is an LP problem with $m n+m+n+q$ variables and $m n+m+r$ constraints. Thus, for problems with not too large number of criteria $(m)$ and preferential weights $(n)$ it can be solved directly. However, similar to the case of minimization of the or-like WOWA, it may be better to deal with the dual of (32) - (36) where $m n$ rows corresponding to variables $d_{i k}$ represent only simple upper bounds. Indeed, while introducing the dual variables: $\mathbf{u}=\left(u_{1}, \ldots, u_{r}\right)$, $\mathbf{v}=\left(v_{1}, \ldots, v_{m}\right)$ and $\mathbf{z}=\left(z_{i k}\right)_{i=1, \ldots, m ; k=1, \ldots, n}$ corresponding to the constraints (33), (34) and (35), respectively, we get the following dual:

$$
\begin{array}{ll} 
& \min \mathbf{u b} \\
\text { s.t. } & \mathbf{u A}-\mathbf{v} \mathbf{C} \geqq \mathbf{0} \\
& v_{i}-\sum_{k=1}^{n} z_{i k}=0 \quad \text { for } i=1, \ldots, m \\
& \sum_{i=1}^{m} z_{i k}=\frac{k}{n} w_{k}^{\prime \prime} \quad \text { for } k=1, \ldots, n \\
0 \leq z_{i k} \leq w_{k}^{\prime \prime} p_{i} & \text { for } i=1, \ldots, m ; k=1, \ldots, n
\end{array}
$$

The dual problem (37), similar to (27), contains $m n+r+m$ variables and $m+n+q$ structural constraints. Therefore, it can be directly solved for quite large values of $m$ and $n$.

\section{Computational Tests}

In order to examine computational performances of the LP models for the WOWA optimization we have solved randomly generated problems with varying 
number $q$ of decision variables and number $m$ of criteria. The core LP feasible set has been defined by a single knapsack-type constraint. Thus, we have analyzed the WOWA maximization problem

$$
\max \left\{A_{\mathbf{w}, \mathbf{p}}(\mathbf{f}(\mathbf{x})): \sum_{j=1}^{q} x_{j}=1, \quad x_{j} \geq 0 \quad \text { for } j=1, \ldots, q\right\}
$$

where $f_{i}(\mathbf{x})=\mathbf{c}_{i} \mathbf{x}=\sum_{j=1}^{q} c_{i j} x_{j}$. Such problems may be interpreted as resource allocation decisions [10].

For our computational tests we have randomly generated problems (38). Coefficients $c_{i j}$ were generated as follows. First, for each $j$ the upper bound $r_{j}$ was generated as a random number uniformly distributed in the interval $[0.05,0.15]$. Next, individual coefficients $c_{i j}$ were generated as uniformly distributed in the interval $\left[-0.75 r_{j}, r_{j}\right]$. In order to generate strictly increasing and positive preference weights $w_{k}$, we generated randomly the corresponding increments $\delta_{k}=w_{k}-w_{k-1}$. The latter were generated as uniformly distributed random values in the range of 1.0 to 2.0 , except from a few ( 5 on average) possibly larger increments ranged from 1.0 to $n / 3$. Importance weights $p_{i}$ were generated according to the exponential smoothing scheme, $p_{i}=\alpha(1-\alpha)^{i-1}$ for $i=1,2, \ldots, m$ and the parameter $\alpha$ is chosen for each test problem size separately to keep the smallest weight $p_{m}$ around 0.001 .

The optimization times were analyzed for various size parameters $m$ and $q$. The basic tests were performed for the standard WOWA model with $n=m$. However, we also analyzed the case of larger $n$ for more detailed preferences modeling, as well as the case of smaller $n$ thus representing a rough preferences model. For each number of decision variables $q$ and number of criteria $m$ we solved 10 randomly generated problems (38). All computations were performed on a PC with the Pentium 4 2.4GHz processor employing the CPLEX 9.1 package. The 600 seconds time limit was used in all the computations.

Table 1. WOWA optimization times [s]: primal model (32)-36]

\begin{tabular}{crrrrrrrr}
\hline $\begin{array}{c}\text { Number of } \\
\text { criteria }(m)\end{array}$ & 10 & 20 & 50 & 100 & 150 & 200 & 300 & 400 \\
\cline { 2 - 9 } & 0.0 & 0.0 & 0.0 & 0.0 & 0.0 & 0.0 & 0.0 & 0.1 \\
10 & 0.1 & 0.1 & 0.0 & 0.1 & 0.1 & 0.1 & 0.1 & 0.1 \\
50 & 1.5 & 2.4 & 3.1 & 4.0 & 4.0 & 4.1 & 3.9 & 4.0 \\
50 & 54.6 & 73.2 & 89.4 & 110.7 & 139.1 & 185.7 & 253.7 & - \\
100 & & &
\end{tabular}

In Tables 1 and 2 we show the solution times for the primal (32)-(36) and the dual (37) forms of the computational model, being the averages of 10 randomly generated problems. Upper index in front of the time value indicates the number of tests among 10 that exceeded the time limit. The empty cell (minus sign) shows that this has occurred for all 10 instances. Both model forms were solved by the CPLEX code with the standard settings. As one can see, the dual form of the 
model performs much better in each tested problem size. It behaves very well with increasing number of variables if the number of criteria does not exceed 100 , and satisfactory if the number of criteria equals 150. Similarly, the model performs very well with increasing number of criteria if only the number of variables does not exceed 50 .

Table 2. WOWA optimization times [s]: dual model (37)

\begin{tabular}{crrrrrrrr}
\hline \multirow{2}{*}{$\begin{array}{c}\text { Number of } \\
\text { criteria }(m)\end{array}$} & \multicolumn{8}{c}{ Number of variables $(q)$} \\
\cline { 2 - 9 } & 10 & 20 & 50 & 100 & 150 & 200 & 300 & 400 \\
\hline 10 & 0.0 & 0.0 & 0.0 & 0.0 & 0.0 & 0.0 & 0.0 & 0.0 \\
20 & 0.0 & 0.0 & 0.0 & 0.0 & 0.0 & 0.0 & 0.0 & 0.1 \\
50 & 0.0 & 0.0 & 0.3 & 0.5 & 0.7 & 0.9 & 1.3 & 1.6 \\
100 & 0.7 & 0.8 & 2.8 & 17.3 & 21.5 & 26.6 & 29.7 & 34.7 \\
150 & 1.8 & 2.6 & 6.0 & 69.0 & 145.9 & 177.5 & 189.0 & 183.3 \\
200 & 4.6 & 6.2 & 13.6 & 179.3 & 395.5 & ${ }^{6} 573.3$ & 8593.2 & - \\
300 & 16.3 & 24.6 & 82.4 & 7473.3 & - & - & - & - \\
400 & 42.7 & 77.5 & 239.6 & - & - & - & - & - \\
\hline
\end{tabular}

In order to examine how much importance weighting of the WOWA complicates our optimization models we have rerun all the tests assuming equal importance weights thus restricting the models to the standard OWA optimization according to [11. Tables 3 and 4 show the solution times for the primal (32) (36) and the dual (37) optimization models, respectively, with equal importance weights while all the other parameters remain generated randomly.

Table 3. OWA optimization times [s]: primal model with equal importance weights

\begin{tabular}{crrrrrrrr}
\hline $\begin{array}{c}\text { Number of } \\
\text { criteria }(m)\end{array}$ & 10 & 20 & 50 & 100 & 150 & 200 & 300 & 400 \\
\cline { 2 - 9 } & 0.0 & 0.0 & 0.0 & 0.0 & 0.0 & 0.0 & 0.0 & 0.0 \\
20 & 0.0 & 0.0 & 0.0 & 0.0 & 0.0 & 0.0 & 0.1 & 0.1 \\
50 & 1.3 & 1.7 & 2.3 & 2.4 & 2.6 & 2.7 & 2.8 & 2.9 \\
100 & 51.2 & 73.4 & 86.6 & 105.9 & 94.4 & 116.6 & 143.6 & 155.5 \\
\hline
\end{tabular}

One may notice that in the case of the primal model the WOWA optimization times (Table 1) are 10-30\% longer than the corresponding OWA optimization times (Table 3). On the other hand, in the case of the dual model the WOWA optimization times (Table 2) turn out to be similar to the corresponding OWA times (Table 4), and frequently even shorter.

Table 5 presents solution times for different numbers of the preferential weights for problems with 100 criteria and 50 variables. One may notice that the computational efficiency can be improved by reducing the number of preferential weights which can be reasonable in non-automated decision making support systems. On the other hand, increasing the number of preferential weights and 
Table 4. OWA Optimization times [s]: dual model with equal importance weights

\begin{tabular}{|c|c|c|c|c|c|c|c|c|}
\hline \multirow{2}{*}{$\begin{array}{l}\text { Number of } \\
\text { criteria }(m)\end{array}$} & \multicolumn{8}{|c|}{ Number of variables $(q)$} \\
\hline & 10 & 20 & 50 & 100 & 150 & 200 & 300 & 400 \\
\hline 10 & 0.0 & 0.0 & 0.1 & 0.0 & 0.0 & 0.0 & 0.0 & 0.0 \\
\hline 20 & 0.0 & 0.0 & 0.0 & 0.1 & 0.0 & 0.0 & 0.0 & 0.1 \\
\hline 50 & 0.1 & 0.2 & 0.3 & 0.7 & 1.0 & 1.5 & 1.6 & 2.3 \\
\hline 100 & 0.7 & 0.9 & 5.0 & 18.0 & 29.6 & 25.7 & 32.5 & 42.1 \\
\hline 150 & 2.2 & 2.8 & 12.6 & 82.3 & 130.5 & 143.9 & 163.2 & 194.8 \\
\hline 200 & 4.8 & 7.8 & 22.4 & 172.2 & 323.6 & ${ }^{2} 452.3$ & ${ }^{4} 505.5$ & ${ }^{9} 586.9$ \\
\hline 300 & 18.8 & 28.9 & 186.8 & ${ }^{9} 549.5$ & - & - & - & - \\
\hline 400 & 44.9 & 96.1 & 417.9 & - & - & - & - & - \\
\hline
\end{tabular}

Table 5. WOWA optimization times $[\mathrm{s}]$ : varying number of preferential weights $(m=$ $100, q=50)$

\begin{tabular}{rrrrrrrrrr}
\hline \multicolumn{10}{c}{ Number of preferential weights $(n)$} \\
3 & 5 & 10 & 20 & 50 & 100 & 150 & 200 & 300 & 400 \\
\hline 0.0 & 0.1 & 0.1 & 0.3 & 2.9 & 2.8 & 1.8 & 2.9 & 5.0 & 7.4 \\
\hline
\end{tabular}

thus the number of breakpoints in the interpolation function does not induce the massive increase in the computational complexity.

\section{Concluding Remarks}

The problem of aggregating multiple criteria to form overall objective functions is of considerable importance in many disciplines. The WOWA aggregation [12] represents a universal tool allowing to take into account both the preferential weights allocated to ordered outcomes and the importance weights allocated to several criteria. The ordering operator used to define the WOWA aggregation is, in general, hard to implement. We have shown that the WOWA aggregations with the monotonic weights can be modeled by introducing auxiliary linear constraints. Exactly, the OWA LP-solvable models introduced in [1] can be expanded to accommodate the importance weighting of the WOWA aggregation.

Our computational experiments have shown that the formulations enable to solve effectively medium size problems. While taking advantages of the dual model the WOWA problems with up to 100 criteria have been solved directly by general purpose LP code within less than half a minute.

\section{References}

1. Damiani, E., De Capitani di Vimercati, S., Samarati, P., Viviani, M.: A WOWAbased aggregation technique on trust values connected to metadata. Electronic Notes Theor. Comp. Sci. 157, 131-142 (2006) 
2. Fodor, J., Roubens, M.: Fuzzy Preference Modelling and Multicriteria Decision Support. Kluwer A.P, Dordrecht (1994)

3. Glover, F., Klingman, D.: The simplex SON method for LP/embedded network problems. Math. Progr. Study 15, 148-176 (1981)

4. Grabisch, M., Orlovski, S.A., Yager, R.R.: Fuzzy aggregation of numerical preferences. Fuzzy sets in decision analysis, operations research and statistics, pp. 31-68. Kluwer AP, Dordrecht (1999)

5. Larsen, H.L.: Importance weighted OWA aggregation of multicriteria queries. In: Proc. North American Fuzzy Info. Proc. Soc. Conf. (NAFIPS'99), pp. 740-744 (1999)

6. Liu, X.: Some properties of the weighted OWA operator. Man and Cyber. B 368, 118-127 (2006)

7. Nettleton, D., Muniz, J.: Processing and representation of meta-data for sleep apnea diagnosis with an artificial intelligence approach. Medical Informatics 63, 77-89 (2001)

8. Ogryczak, W.: On Goal Programming Formulations of the Reference Point Method. J. Opnl Res. Soc. 52, 691-698 (2001)

9. Ogryczak, W.: Multiple criteria optimization and decisions under risk. Control \& Cyber 31, 975-1003 (2002)

10. Ogryczak, W., Śliwiński, T.: On equitable approaches to resource allocation problems: the conditional minimax solution, J. Telecom. Info. Tech. 3/02, 40-48 (2002)

11. Ogryczak, W., Śliwiński, T.: On solving linear programs with the ordered weighted averaging objective. Eur. J. Opnl. Res. 148, 80-91 (2003)

12. Torra, V.: The weighted OWA operator. Int. J. Intell. Syst. 12, 153-166 (1997)

13. Torra, V.: The WOWA operator and the interpolation function $\mathrm{W}^{*}$ : Chen and Otto's interpolation method revisited. Fuzzy Sets Syst. 113, 389-396 (2000)

14. Valls, A., Torra, V.: Using classification as an aggregation tool for MCDM. Fuzzy Sets Syst. 115, 159-168 (2000)

15. Yager, R.R.: On ordered weighted averaging aggregation operators in multicriteria decision making. IEEE Trans. Systems, Man and Cyber. 18, 183-190 (1988)

16. Yager, R.R.: Constrained OWA aggregation. Fuzzy Sets Syst. 81, 89-101 (1996)

17. Yager, R.R.: Quantifier guided aggregation using OWA operators. Int. J. Intell. Syst. 11, 49-73 (1996)

18. Yager, R.R.: Including Importances in OWA Aggegations Using Fuzzy Systems Modeling. IEEE Trans. Fuzz. Syst. 6, 286-294 (1998)

19. Yager, R.R., Filev, D.P.: Essentials of Fuzzy Modeling and Control. Wiley, New York (1994)

20. Yager, R.R., Kacprzyk, J.: The Ordered Weighted Averaging Operators: Theory and Applications. Kluwer AP, Dordrecht (1997) 